Knie J. 2020 2:235-236

https://doi.org/10.1007/s43205-020-00077-6

(c) Springer Medizin Verlag GmbH, ein Teil von Springer Nature 2020

\author{
Philipp von Roth ${ }^{1}$ Heiko Graichen ${ }^{2}$ Tilman Pfitzner ${ }^{3}$ \\ ${ }^{1}$ sporthopaedicum, Straubing, Deutschland \\ ${ }^{2}$ Asklepios Klinik Lindenlohe GmbH, Schwandorf, Deutschland \\ ${ }^{3}$ Klinik für Endoprothetik, Knie- und Hüftchirurgie, Department für Bewegungschirurgie, Vivantes \\ Klinikum Berlin, Berlin, Deutschland
}

\title{
Neues aus der Endoprothetik
}

Liebe Kolleginnen und Kollegen,

auch wenn in der aktuellen Zeit natürlich andere Themen unser aller Alltag beherrschen, so müssen wir den Blick trotzdem auch in die Zukunft unseres Bereiches richten und uns kritisch mit den Ergebnissen und Neuerungen auseinandersetzen.

In nur wenigen Bereichen der Medizin ist technologischer Fortschritt und Digitalisierung so eng mit der operativen Therapie verknüpft wie in der Knieendoprothetik. Daher verwundert es nicht, dass sich im Lauf der letzten Jahrzehnte Innovationen und technische Weiterentwicklungen besonders in diesem Bereich gezeigt haben und immer wieder Teil der wissenschaftlichen Diskussion gewesen sind.

Dies betrifft zum einen die Evolution der Implantate selbst. Hier ist sowohl im Bereich der Implantatdesigns als auch der Implantatwerkstoffe der Versuch unternommen worden, eine Verbesserung der Funktion, Biomechanik und Haltbarkeit zu erreichen. Als gutes Beispiel ermöglichen aktuelle Polyethylene aller Voraussicht nach auch langfristig eine höhere Belastung, ohne die teils aus der Vergangenheit bekannten starken Verschleißbilder.

Zum anderen zeigt sich ein deutlicher Innovationstrend im Bereich der operativen Assistenzsysteme und Technologien, die dem Operateur eine bessere Umsetzung der OP-Planung ermöglichen sollen.

Als dritter Bereich ist die Prozessoptimierung des Behandlungsablaufes ebenfalls in den Fokus der Diskussion gelangt. Auch durch Standardisierung und Optimierung der Prozesse scheinen eine Ver- besserung des Ergebnisses und Senkung der Komplikationsraten möglich.

Anders als in anderen Bereichen, in denen die Technisierung und Digitalisierung oft subjektiv einen stetig fortschreitenden Prozess darstellen, zeigt sich dies im Bereich der Knieendoprothetik deutlich undulierender: Neue Technologien halten Einzug, werden stark beworben und verschwinden bereits nach relativ kurzer Zeit wieder. Häufig zeigt sich nach deren Optimierung oder einfach einergewissen Zeit eine Renaissance mit einem erneuten Versuch der Implementierung.

Der größte Kritikpunkt dieser technologischen Innovationen ist, dass in den letzten Jahren keine der gängigen Innovation eine relevant messbare Verbesserung der Patientenzufriedenheit wissenschaftlich belegen konnte. Daher steht im Fokus der gegenwärtigen Diskussion nicht alleine die technische Umsetzung der etablierten „konventionellen OP-Techniken“, sondern neue Strategien der Prozessoptimierung und der Implantationsphilosophie, um eine Verbesserung der Patientenzufriedenheit $\mathrm{zu}$ erreichen. Hier ist die richtige Alignmentstrategie in der Koronarebene in den letzten Jahren sicher am meisten in der - oft auch kontroversen Diskussion gewesen.

Diese neuen Konzepte der Ausrichtung erscheinen, da sie sich mitunter im Grenzbereich der bislang akzeptierten Implantatpositionen bewegen, weniger fehlerverzeihend. Folglich fordern sie von uns als Operateur eine sehr präzise intraoperative Umsetzung. Hier bekommen Assistenzsysteme, wie Navigation oder Robotik, auf einmal eine neue und fundamental wichtige Bedeutung. Während diese Techno- logien zwar bislang keine eindeutige Verbesserung des klinischen Ergebnisses gezeigt haben, konnten sie jedoch zweifelsfrei wissenschaftlich belegen, dass sie eine Steigerung der Präzision in der Implantatausrichtung und somit Umsetzung der präoperativen Planung ermöglichen. Damit stellen sie zukünftig vermutlich den Schlüssel zu einer sicheren und effizienten Umsetzung neuerer Alignmentstrategien dar.

In diesem Themenheft beschäftigen wir und sowohl mit den neuen technologischen Möglichkeiten als auch mit den neuen Philosophien der Implantatausrichtung.

Um diese Bereiche richtig einordnen zu können, ist es jedoch von essenzieller Bedeutung, zu verstehen, wo die Probleme und Revisionsursachen in der Knieendoprothetik in der Gegenwart liegen. Daher gehen wir zuerst einen Schritt zurück und betrachten die aktuellen Versagensursachen. In den Artikeln von Arnd Steinbrück und Eric Röhner werden die Revisionsursachen der unikondylären Endoprothetik und der Totalendoprothetik analysiert, um ein besseres Verständnis der Problemfelder zu entwickeln und Potenziale für technologische Weiterentwicklung $\mathrm{zu}$ identifizieren.

In den weiteren Beiträgen widmen wir uns den Feldern der aktuellen Innovationen. Lisa Renner gibt einen Überblick über den derzeitigen Stand der digitalen Prozessoptimierung, die damit verbundenen Vorteile und mögliche Limitationen. Dies wird uns insbesondere unterdem Gesichtspunkt der zunehmenden Ökonomisierung sicher in den nächsten Jahren als wichtiges Thema begleiten und eine differenzierte Diskussion erforder- 
lich machen. Die bereits oben genannten in der aktuellen Diskussion omnipräsenten Technologien der Assistenzsyteme und Ausrichtungsphilosophien werden in den Beiträgen von Max Ettinger und Tilman Calliess dargestellt. Max Ettinger setzt den Schwerpunkt dabei auf die Darstellung der verschiedenen Ausrichtungsphilosophien und die Optionen der technischen Umsetzung. Tilman Calliess zeigt dagegen die Anwendungsbereiche für die unikondyläre Endoprothetik und das Zusammenspiel aus Optimierung der Position und Möglichkeiten der Einbeziehung des Weichteilbalancing.

Wir hoffen, Ihnen mit diesem Themenheft des Knie Journals einen umfassenden Überblick über die aktuellen Probleme und Versagensursachen der Knieendoprothetik geben zu können und Ihnen zu zeigen, in welchen Bereichen die aktuellen technologischen Innovationen stattfinden und inwieweit deren Umsetzung bereits Eingang in die tägliche Klinik gefunden hat.

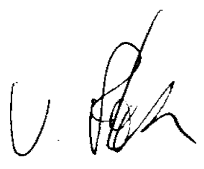

PD Dr. Philipp von Roth

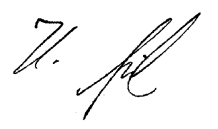

Prof. Dr. Heiko Graichen<smiles>C=C1C=CC(CCC)C1CCC</smiles>

PD Dr. Tilman Pfitzner

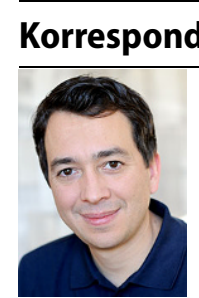

sporthopaedicum

Bahnhofplatz 27,

94315 Straubing,

Deutschland

roth@sporthopaedicum.de

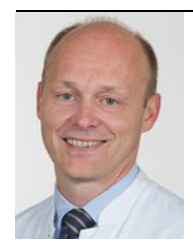

Prof. Dr. Heiko Graichen Asklepios Klinik Lindenlohe $\mathrm{GmbH}$

Lindenlohe 18,

92421 Schwandorf,

Deutschland

h.graichen@asklepios.com

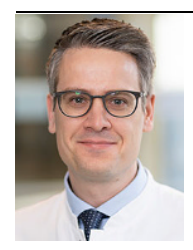

PD Dr. Tilman Pfitzner

Klinik für Endoprothetik, Knie- und Hüftchirurgie, Department für Bewegungschirurgie, Vivantes Klinikum Berlin

Neue Bergstr. 6, 13585 Berlin, Deutschland

tilman.pfitzner@vivantes.de

Interessenkonflikt. P. von Roth, H. Graichen und T. Pfitzner geben an, dass kein Interessenkonflikt besteht.

\section{Der implantierte Code}

Produktkennzeichnung für jahrzehntelange Nachverfolgung entwickelt

Mit Codes, die unsichtbar im Inneren von Implantaten liegen, kann die Fertigung 3-D-gedruckter Produkte effizienter gestaltet und Produktpiraterie besser verhindert werden. Angewendet in der Medizintechnik, sind damit künstliche Gelenke und andere Implantate in Zukunft über ihre gesamte Lebensdauer hinweg eindeutig identifizierbar.

Schulter, Hüfte, Knie - wer heute ein Implantat trägt, hat auch einen Implantatpass bei sich. Er gibt Auskunft über die genaue Bezeichnung, die Seriennummer und den Herstellenden des Implantats. Verlässlicher und sicherer lassen sich Implantate womöglich bald mit einem neuen Verfahren identifizieren, das gerade am FraunhoferInstitut für Werkzeugmaschinen und Umformtechnik (IWU) erprobt wird: Beim 3-D-Druck künstlicher Gelenke werden im Inneren der Bauteile kleinste Hohlräume als Codes eingearbeitet. Sie sind unveränderbar und können jederzeit von Röntgengeräten oder mit einer Computertomographie eindeutig erkannt werden.

Ärztinnen und Ärzte können so im Notfall mit einem Blick in eine Datenbank schnell herausfinden, welche Eigenschaften ein Implantat hat - auch wenn Patienten den Pass nicht bei sich tragen oder nach vielen Jahren verloren haben.

Quelle: Fraunhofer-Institut für Werkzeugmaschinen und Umformtechnik (IWU), www.iwu.fraunhofer.de 